\author{
UNITED STATES DEPARTMENT OF THE INTERIOR \\ GEOLOGICAL SURVEY
}

\title{
Computer and Hand-calculator Programs to Determine Extension or Contraction of Faulted Marker Planes
}

$$
\begin{aligned}
& \text { P. T. Delaney } 1 \\
& \text { M. D. Jackson } 2
\end{aligned}
$$

$$
\text { Open-file Report 85-107 }
$$

This report is preliminary and has not been reviewed for conformity with U.S. Geological Survey editorial standards and stratigraphic nomenclature.

1 U.S. Geological Survey 2255 N. Gemini Dr. Flagstaff, Arizona, 86001
2 Department of Earth and Planetary Sciences Johns Hopkins University Baltimore, Maryland, 21218 


\begin{abstract}
AB S TRACT
Programs are presented which calculate angular relations among fault attitude, marker-plane attitude and slip direction, and determine the extension or contraction of the marker by the fault slip. The programs are written both in FORTRAN and in the reverse-Polish language implemented on Hewlett-Packard type hand calculators. Examples of program use are presented.
\end{abstract}

\title{
INTRODUCTION
}

It has been shown by Jackson and Delaney (1985) that certain angular relations among a marker plane, a fault and the direction of fault slip provide a measure of the extensional or contractional separation of a marker plane. These angles are: $\phi$, the angle between the downward directed normals to the fault and marker planes; $\theta$, the angle from the intersection of the fault and marker planes to the slip direction; and $\omega$, the angle between the direction of maximum contraction and the direction of slip. The first two or the last of these angles can be used to ascertain the extension or contraction of the marker plane in a direction normal to the line of intersection of the fault and marker. This paper presents a program written in FORTRAN for computers, and a program written in a reverse-Polish language for HewlettPackard calculators, to calculate these angular relations.

The method of calculating the angle $\theta$ from the null to the slip directions used by Jackson and Delaney (1985) can result in divide-by-zero conditions. This situation is treated by calculating the magnitude of $\theta$ from the dot-product relation $\cos |\theta|=|\vec{N} \cdot \vec{S}|$, where $\vec{N}$ and $\vec{S}$ are the null and slip vectors, respectively, and the vertical bars denote absolute values of the 
enclosed quantity. The sign of the angle $\theta$ is positive if, looking at the hanging-wall surface of the fault, the angle from the intersection with the marker to the slip direction is drawn in a clockwise direction. This is equivalent to finding whether the direction given by the cross product relation $\vec{N} \times \vec{S}$ is in the same direction (positive $\theta$ ) or opposite direction (negative $\theta$ ) as the downwarded directed normal to the fault plane, $\vec{F}$.

\section{THE FORTRAN PROGRAM}

The FORTRAN program employs the "structured branching options" (see Meissener and Organick, 1980, p. 480) available in ANSI FORTRAN 77 implementations. The program is thoroughly documented and follows the same notation as used by Jackson and Delaney (1985). The program (called FAULT) calls six subroutines: DDDTDC, which converts dip-direction and dip data to direction-cosine data; TPTDC, which converts trend and plunge data to direction-cosine data; DCTTP, which converts direction-cosine data to trend and plunge data; CROSS, which computes the vector cross product; NORM, which normalizes a vector to unit length; and DOT, which computes the vector dot product. The program prompts the user for input at the keyboard, and displays output on a CRT or line printer. It is therefore assumed that the standard input and output devices are interactive, and denoted by the logical unit numbers 5 and 6, respectively. Listings are given in Appendix A.

It is assumed that no field measurement is more accurate than $1^{\circ}$; if the angle $\phi$ is within $1^{\circ}$ of $90^{\circ}$ or the angle $\theta$ is within $1^{\circ}$ of $0^{\circ}$, then the fault slip is identified as "null"-- it neither extended nor contracted the marker. In addition, the program calculates the angle between the slip direction and the fault plane. By definition, this angle should be zero. If it is found to 
be greater than $1^{\circ}$, it is used to determine the limits of null faulting.

The example below illustrates the use of the FORTRAN program. Usersupplied entries are underlined.

RUN FAULT

MARKER: Dip Direction and dip $(\operatorname{deg})=? ?=$ $317 \quad 20$

FAULT: Dip Direction and Dip (deg) = ?? =

18045

SLICKENLINE: Trend and Plunge $(\mathrm{deg})=? ?=$ 922

OFFSET: Hanging Wall UP (U), DOWN (D),

$\underline{\mathrm{D}}$ or NO (N) apparent offset $=?$ ? =

92. 2. = Trend, Plunge of Hanging Wall SLIP DIRECTION

$0 .=$ Eta, ANGLE of the SLIP VECTOR from the FAULT PLANE

61. = Phi, ANGLE between MARKER and FAULT PLANES

101. 11. = Trend, Plunge of NILL DIRECTION

-18. = Theta, ANGLE from NULL to SLIP DIRECTION

99. = Omega, ANGLE from PURE-CONTRACTION DIRECTION to SLIP DIRECTION EXTENSION FAULT

Another Fault ?? ( $\mathrm{Y} / \mathrm{N})$

Y

MARKER PLANE: Dip Direction and Dip $=317.20$.

FAULT: Dip Direction and Dip $(\mathrm{deg})=$ ? =

$180 \quad 45$

SLICKENLINE: Trend and Plunge (deg) = ?? = 922

OFFSET: Hanging Wall UP (U), DOWN (D), or NO (N) apparent offset = ?? =

$\underline{\mathrm{U}}$

272. -2 . = Trend, Plunge of Hanging Wall SLIP DIRECTION

$0 .=$ Eta, ANGLE of the SLIP VECTOR from the FAULT PLANE

61. = Ph1, ANGLE between MARKER and FAULT PLANES

101. 11. = Trend, Plunge of NULL DIRECTION

18. = Theta, ANGLE from NULL to SLIP DIRECTION

81. = Omega, ANGLE from PURE-CONTRACTION DIRECTION to SLIP DIRECTION 


\section{CONTRACTION FAULT}

Another Fault ?? ( $\mathrm{Y} / \mathrm{N})$

N

Another Marker?? ( $\mathrm{Y} / \mathrm{N})$

$\underline{\mathrm{Y}}$

13570

MARKER: Dip Direction and $\operatorname{dip}(\operatorname{deg})=? ?=$ $180 \quad 45$

FAULT: Dip Direction and Dip (deg) = ?? =

SLICKENLINF: Trend and Plunge $(\mathrm{deg})=$ ?? = 922

OFFSET: Hanging Wall UP (U), DOWN (D), or NO (N) apparent offset = ?? =

$\underline{\mathrm{D}}$

92. 2. = Trend, Plunge of Hanging Wall SLIP DIRECTION

$0 .=$ Eta, ANGLE of the SLIP VECTOR from the FAULT PLANE

45. = Phi, ANGLE between MARKFR and FAULT PLANES

26. -42 . = Trend, Plunge of NULL DIRECTION

206. $42 .=$ in Lower Hemisphere

74. = Theta, ANGLE from NULL to SLIP DIRECTION

47. = Omega, ANGLE from PURE-CONTRACTION DIRECTION to SLIP DIRECTION

CONTRACTION FAULT

Another Fault ?? ( $\mathrm{Y} / \mathrm{N})$

$\mathrm{N}$

Another Marker?? ( $\mathrm{Y} / \mathrm{N})$

$\underline{Y}$

MARKER: Dip Direction and dip $(\operatorname{deg})=? ?=$

$45 \quad 45$

FAULT: Dip Direction and Dip (deg) = ?? =

$225 \quad 45$

SLICKENLINE: Trend and Plunge (deg) = ?? = 22545

OFFSET: Hanging Wa11 UP (U), DOWN (D), or NO $(\mathrm{N})$ apparent offset $=$ ?? =

$\underline{\mathrm{D}}$

225. 45. = Trend, Plunge of Hanging Wall SLIP DIRECTION

$0 .=$ Eta, ANGLE of the SLIP VECTOR from the FAULT PLANE

90. = Phi, ANGLE between MARKER and FAULT PLANES

-45. $0 .=$ Trend, Plunge of NULL DIRECTION

135. $0_{0}=$ in Lower Hemisphere 


\section{HAND-CALCULATOR PROGRAM}

The calculation, written for a Hewlett-Packard 15-C calculator, requires 20 storage registers and about 200 lines of instructions. Parameters stored in the registers are shown in Table 1. A listing is given in Appendix B. For brevity, only the angle $\phi$ between the downward-directed marker- and faultplane normals, and the angle $\theta$ from the null direction to the slip direction, are calculated. Extension or contraction can be determined from these angles (Table 2). There are five programs and three subroutines. The first program uses the dip direction and dip of the marker plane to calculate and store the direction cosines of the downward-directed normals to that plane. The second program performs the same task for the fault plane. For faults with hangingwa11-down displacements, the third program uses the trend and plunge of the

TABLE 1: Contents of storage registers

REGISTER \#

0

1

2

3

4

5

6

7

8

9

.0

$.1-.9$

\section{CONTENTS}

$1^{\text {marker }}$

$\mathrm{m}^{\text {marker }}$

$\mathrm{n}^{\text {marker }}$

$1^{\text {fault }}$

$\mathrm{m}^{\text {fault }}$

$\mathrm{n}^{\text {fault }}$

${ }_{1}^{\text {slip }}$

$\mathrm{m}^{\mathrm{s} 11 \mathrm{p}}$

$\mathrm{n}^{\mathrm{s} 1 \mathrm{p}}$

$\phi$, angle between downward-directed marker- and fault-normals

$\theta$, angle from null to slip direction

miscellaneous
$1, \mathrm{~m}, \mathrm{n}$ are cosines of angles that

superscripted vector makes with

the South, East and Up directions. 
Table 2: Extension or Contraction from Angles $\phi$ and $\theta$

\begin{tabular}{cccc} 
& $0^{\circ} \leqslant \phi<90^{\circ}$ & $\phi=0^{\circ}$ & $90^{\circ}<\phi \leqslant 180^{\circ}$ \\
\hline$-90^{\circ} \leqslant \theta<0^{\circ}$ & Extend & Nu11 & Contract \\
$0^{\circ}=\theta$ & Nul1 & Nu11 & Nul1 \\
$0^{\circ}<\theta \leqslant 90^{\circ}$ & Contract & Nu11 & Extend
\end{tabular}

slip direction to calculate and store the equivalent direction cosines. For faults with hanging-wa11-up displacements, the fourth program performs the same calculation as the third. The fifth program calculates, displays and stores the angles $\phi$ and $\theta$.

In the following, the instructions, input keystrokes and the output are shown for the first and second examples given above. Underlined numbers refer to the values of the parameters for the particular example.

\section{STEP INSTRUCTIONS INPUT KEYSTROKE DISPLAY}

1. Input marker-plane data

a. Marker dip direction

(clockwise degrees from North) 317

b. Marker dip

(degrees down from horizontal) 20

c. Run program A $\mathrm{f} \mathrm{A}$

2. Input fault-plane data

a. $\frac{\text { Fault dip direction }}{\text { (clockwise degrees from North) } 180}$

d. Fault dip

(degrees down from horizontal) 45

c. Run program B f B

3. Input slip-direction data
a. $\frac{\text { Slickenline trend }}{\text { (clockwise degrees }}$
from North)
$\underline{92}$
ENTER
b. Slickenline plunge
(degrees down from horizontal) 2

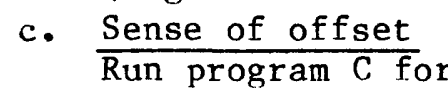
hanging wal1 down displacements
f C 
4. Calculate $\phi$ and $\theta$

Run program E

$$
\text { f } E
$$

$$
\begin{array}{r}
61(=\phi) \\
-18(=\theta)
\end{array}
$$

For the same marker-plane and fault-plane attitudes, it is not necessary to re-enter these orientations to enter a different slickenline. Similarily, it is not necessary to re-enter the attitude of the marker for new fault and slickenline attitudes. For hanging-wall-up fault slips program $D$ is run instead of program $\mathrm{C}$ :

$\begin{array}{lll}\text { STEP INSTRUCTIONS INPUT KEYSTROKE DISPLAY } & \end{array}$

5. Input slip-direction data
a. Slickenline trend
(clockwise degrees from North) $92 \quad$ ENTER.
b. Slickenline plunge
(degrees down from horizontal) $\underline{2}$
c. Sense of offset
Run program D for
hanging wall up displacements f D
6. Calculate $\phi$ and $\theta$
Run program $\mathrm{E}$
f $\mathrm{E}$
$61(=\phi)$
$18(=\theta)$

\section{REF ERENCES}

Jackson, M.D., and P.T. Delaney, 1985, Extension and contraction of faulted marker planes: Geology, to be submitted. 16 ms. pages, 5 figures, 2 tables.

Meissner, L.P., and E.I. Organick, 1980, Fortran 77: Featuring Structured Programming, Addison-Wesley, Reading, Mass., 500 p. 


\section{APPENDIX A: FORTRAN PROGRAM}

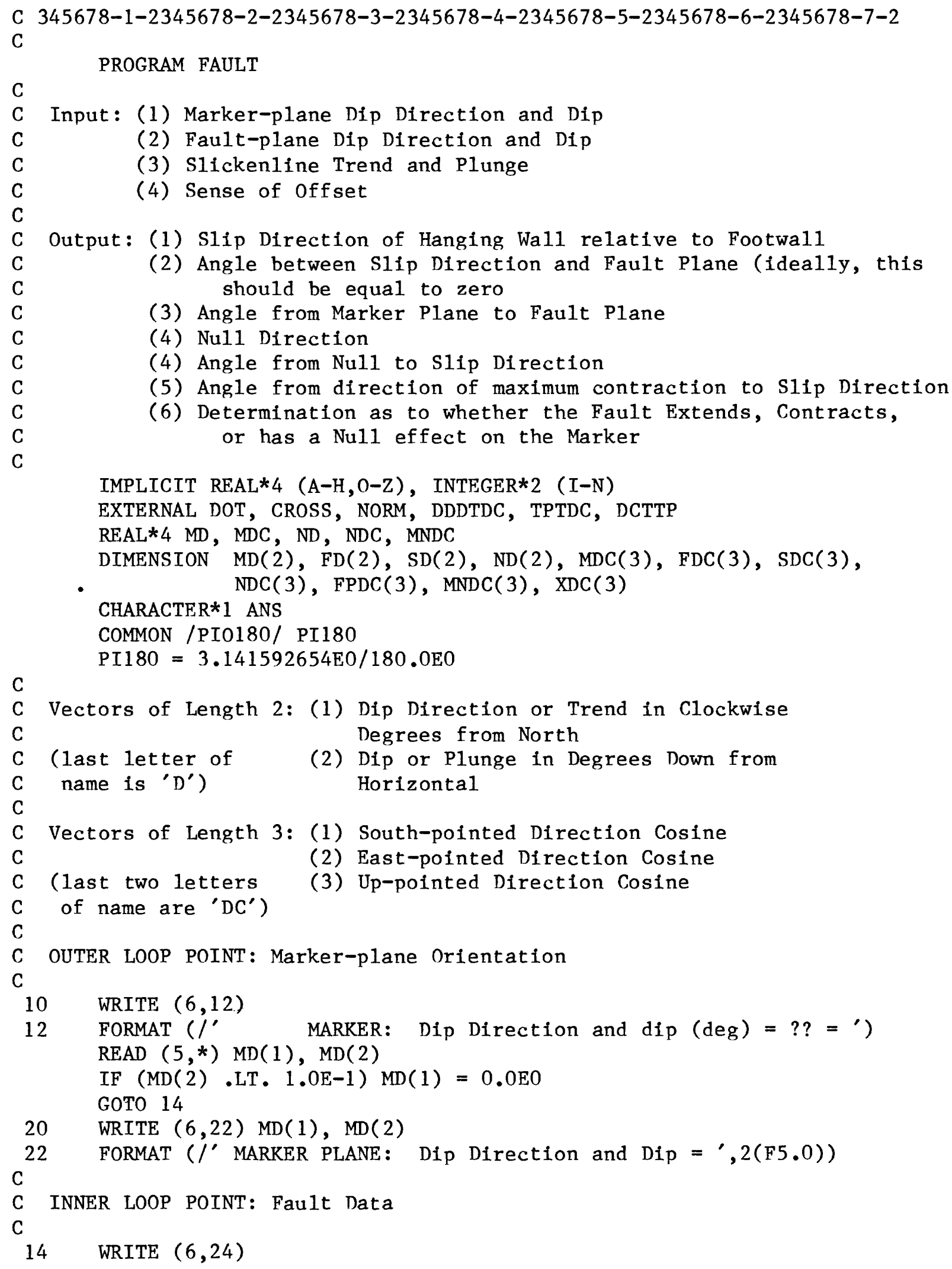




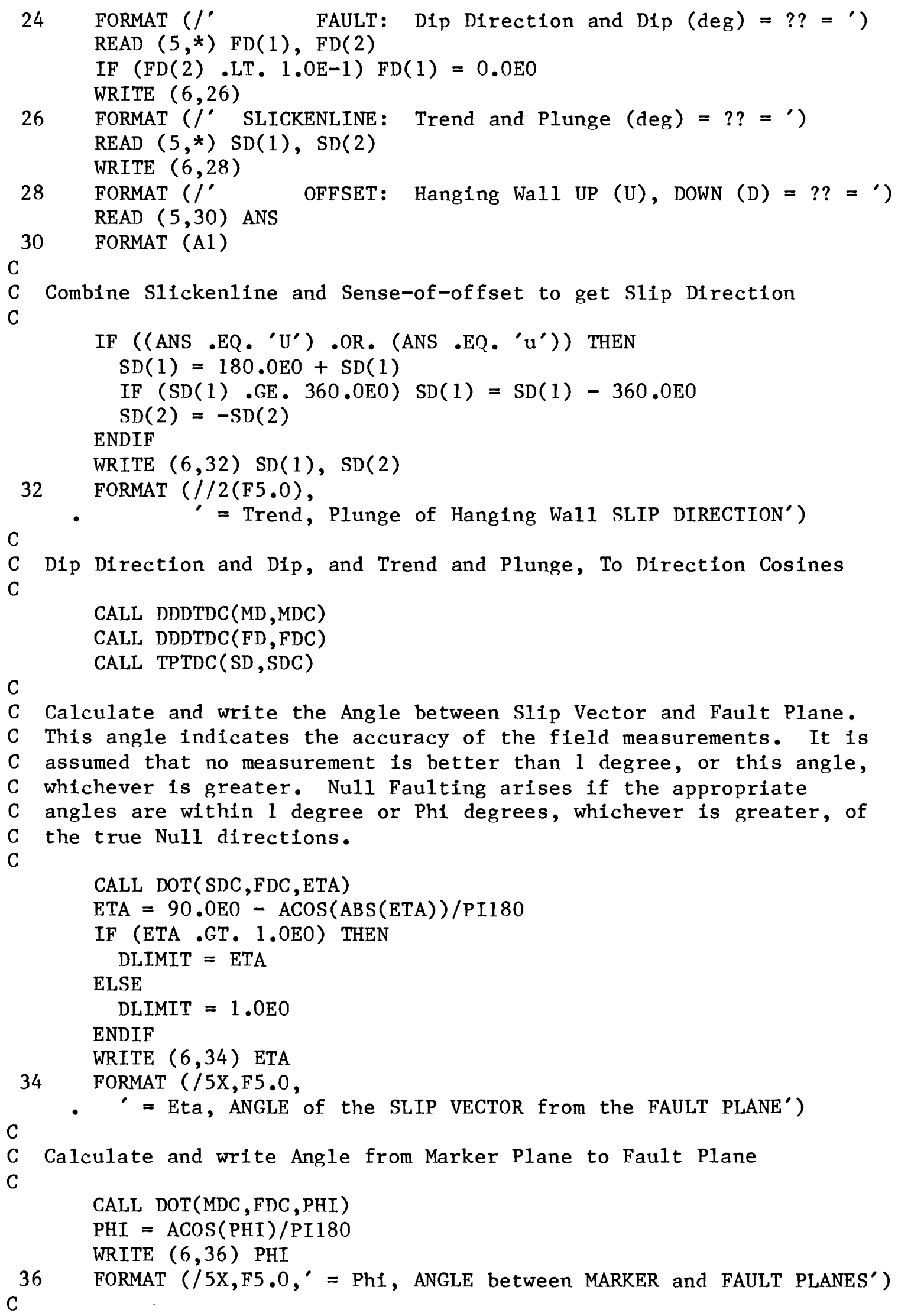


C

CaIculate and write Null direction

CALL CROSS (FDC,MDC, NDC)

CALL NORM(NDC)

CALL DCTTP(NDC, ND)

WRITE $(6,38)$ ND(1), ND(2)

38

FORMAT (/2(F5.0), $)^{\prime}=$ Trend, Plunge of NULL DIRECTION')

IF (ND(2) .LT . 0.0E0) THEN

$\mathrm{ND}(1)=\mathrm{ND}(1)+180.0 \mathrm{E} 0$

$\mathrm{ND}(2)=-\mathrm{ND}(2)$

IF (ND(1) .GE. 360.0E0) $\mathrm{ND}(1)=\mathrm{ND}(1)-360.0 \mathrm{E} 0$

WRITE $(6,40)$ ND( 1$), \mathrm{ND}(2)$

40

FORMAT (2(F5.0), = ENDIF

C

C Calculate and write Theta Angle

$C$ The method used below is more cumbersome, but more robust, than that

$C$ described in the paper--there $s$ no possibility for divide-by-zero

$\mathrm{C}$ errors. The two methods are entirely equivalent.

$\mathrm{C}$

CALL DOT(NDC, SDC, THETA)

THETA $=\operatorname{ACOS}($ ABS $($ THETA $)) /$ PI 180

IF (THETA .GT. DLIMIT) THEN

CALL CROSS (NDC, SDC,FPDC)

CALL NORM(FPDC)

CALL DOT(FDC,FPDC, TEST)

IF (TEST .LT . 0.0E0) THETA = -THETA

ENDIF

WRITE $(6,50)$ THETA

50

FORMAT $(/ 5 \mathrm{X}, \mathrm{F} 5.0$,

C

C Calculate and write Omega angle

C

CALL CROSS (MDC, NDC, XDC)

CALL NORM(XDC)

CALL DOT ( SDC, XDC, OMEGA)

OMEGA $=\operatorname{ACOS}($ OMEGA) $/ \mathrm{PI} 180$

WRITE $(6,60)$ OMEGA

60 FORMAT ( $/ 5 \mathrm{X}, \mathrm{F} 5.0)^{\prime}=$ Omega, ANGLE from PURE-CONTRACTION ', - 'DIRECTION to SLIP DIRECTION')

C

$\mathrm{C}$

C

C If Phi $<90 \mathrm{deg}, 0 \mathrm{deg}<$ Theta $<90 \mathrm{deg}-$ Extension Fault

C 0 deg $>$ Theta $>-90 \mathrm{deg}-$ - Contraction Fault

C If Phi $>90 \mathrm{deg}, 0 \mathrm{deg}>$ Theta $>90 \mathrm{deg}--$ Extension Fault

C 0 deg $<$ Theta $<-90 \mathrm{deg}-$ Contraction Fault

C

C If Phi $=90 \mathrm{deg}$ or Theta $=0$ deg -- Null Fault

C 


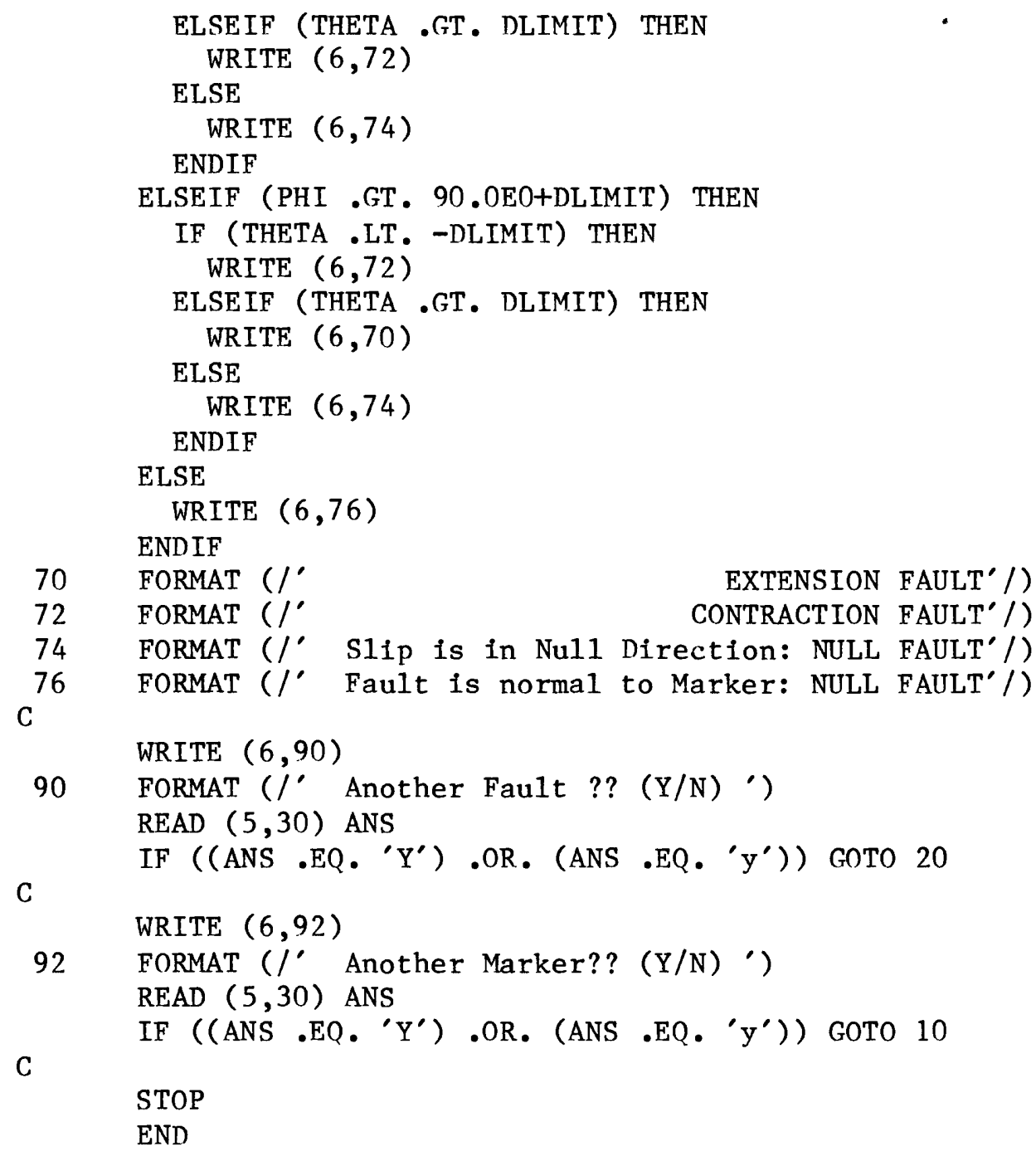

C

$\mathrm{DC}(1)=\operatorname{SIN}(\mathrm{PI} 180 * \mathrm{D}(2)) * \operatorname{COS}(\mathrm{PI} 180 * \mathrm{D}(1))$

$\mathrm{DC}(2)=-\operatorname{SIN}(P I 180 * \mathrm{D}(2)) * \operatorname{SIN}(\operatorname{PI} 180 * \mathrm{D}(1))$

$\mathrm{DC}(3)=-\operatorname{CoS}(\mathrm{PI} 180 * \mathrm{D}(2))$

C

RETURN

END 
C $345678-1-2345678-2-2345678-3-2345678-4-2345678-5-2345678-6-2345678-7-2$

$\mathrm{C}$

SUBROUTINE TPTDC(D,DC)

$\mathrm{C}$

C Napier's rules for spherical triangles to convert from Trend and

C Plunge To Direction Cosines

C

IMPLICIT REAL*4 (A-H,O-Z), INTEGER*2 (I-N)

DIMENSION $\mathrm{D}(2), \mathrm{DC}(3)$

COMMON /PIO180/ PI180

C

$$
\begin{aligned}
& \mathrm{DC}(1)=-\operatorname{Cos}(\mathrm{PI} 180 * \mathrm{D}(1)) * \operatorname{Cos}(\mathrm{PI} 180 * \mathrm{D}(2)) \\
& \mathrm{DC}(2)=\operatorname{Cos}(\mathrm{PI} 180 * \mathrm{D}(2)) * \operatorname{SIN}(\mathrm{PI} 180 * \mathrm{D}(1)) \\
& \mathrm{DC}(3)=-\operatorname{SIN}(\operatorname{PI} 180 * \mathrm{D}(2))
\end{aligned}
$$

C

RETURN

END

C 345678-1-2345678-2-2345678-3-2345678-4-2345678-5-2345678-6-2345678-7-2

C

SUBROUTINE DCTTP(DC,D)

C

C Napier's rules for spherical triangles to convert from Direction

C Cosines for a downward pointed plane normal To Trend and Plunge

$\mathrm{C}$

IMPLICIT REAL*4 (A-H,O-Z), INTEGER*2 (I-N)

DIMENSION D(2), DC( 3 )

COMMON /PIO180/ PI180

C

$D(2)=\operatorname{ASIN}(-D C(3)) / P I 180$

$A=\cos (P I 180 * D(2))$

$B=\operatorname{ASIN}(D C(2) / A) / P I 180$

IF (B .GT. 0.0E0) THEN

$D(1)=A \operatorname{Cos}(-D C(1) / A) / P I 180$

ELSE

$D(1)=-A \operatorname{Cos}(-D C(1) / A) / P I 180$

ENDIF

C

RETURN

END 
C 345678-1-2345678-2-2345678-3-2345678-4-2345678-5-2345678-6-2345678-7-2

C

SUBROUTINE CROSS(A,B,Z)

C

C vector CROSS product

$\mathrm{C}$

IMPLICIT REAL*4 (A-H,O-Z), INTEGER*2 (I-N)

DIMENSION $\mathrm{A}(3), \mathrm{B}(3), \mathrm{Z}(3)$

$\mathrm{C}$

$$
\begin{aligned}
& \mathrm{Z}(1)=A(2) * \mathrm{~B}(3)-\mathrm{B}(2) * A(3) \\
& \mathrm{Z}(2)=-\mathrm{A}(1) * \mathrm{~B}(3)+\mathrm{B}(1) * A(3) \\
& \mathrm{Z}(3)=A(1) * \mathrm{~B}(2)-\mathrm{B}(1) * A(2)
\end{aligned}
$$

C

RETURN

END

C 345678-1-2345678-2-2345678-3-2345678-4-2345678-5-2345678-6-2345678-7-2

C SUBROUTINE NORM(Z)

C

C NORMalize a vector to unit length

C

IMPLICIT REAL*4 (A-H, O-Z), INTEGER*2 (I-N)

EXTERNAL DOT

DIMENSION Z(3)

C

CALL DOT $(Z, Z, Z M A G)$

ZMAG $=\mathrm{SQRT}(Z M A G)$

$Z(1)=Z(1) / Z M A G$

$Z(2)=Z(2) / Z M A G$

C

$Z(3)=Z(3) / Z M A G$

RETURN

END

C $345678-1-2345678-2-2345678-3-2345678-4-2345678-5-2345678-6-2345678-7-2$

C

SUBROUTINE DOT $(A, B, Z)$

C

C vector DOT product

C

IMPLICIT REAL*4 (A-H, O-Z), INTEGER*2 (I-N)

DIMENSION $\mathrm{A}(3), \mathrm{B}(3)$

C

$\mathrm{Z}=\mathrm{A}(1) * \mathrm{~B}(1)+\mathrm{A}(2) * \mathrm{~B}(2)+\mathrm{A}(3) * \mathrm{~B}(3)$

C

RFTURN

END 


\section{APPENDIX B: CALCULATOR PROGRAM}

STEP KEY ENTRY KEY CODE COMMENTS

1 f 1 b1 a 422111 Program A: direction cosines, 1, m and $n$, of downward pointed marker-plane normal, M, calculated from dip direction and dip.

$\begin{array}{lllll}2 & \text { gsb } 1 & 32 & 1 & \text { Go to subroutine } 1 \\ 3 & \text { sto } 2 & 44 & 2 & \text { Store } \mathrm{n}^{\mathrm{M}} \text { in register } 2 \\ 4 & \text { r } \downarrow & 33 & \text { Ro11 } \mathrm{n}^{\mathrm{M}} \text { off stack } \\ 5 & \text { sto } 1 & 44 & \text { Store } \mathrm{m}^{\mathrm{M}} \text { in register } 1 \\ 6 & \text { r } & 33 & \text { Rol1 } \mathrm{m}^{\mathrm{M}} \text { off stack } \\ 7 & \text { sto } 0 & 440 & \text { Store } 1^{\mathrm{M}} \text { in register } 0 \\ 8 & \text { g rtn } & 43 & 32 & \text { Return -- end of program }\end{array}$

9 f 1 b1 b 422112 Program B: direction cosines, 1 , m and $n$, of downward pointed fault-plane normal, F, calculated from dip direction and dip.

10 gsb $1 \quad 321 \quad$ Go to subroutine 1

11 sto $5 \quad 445$ Store $\mathrm{n}^{\mathrm{F}}$ in register 5

$12 \mathrm{r} \downarrow \quad 33 \quad$ Rol1 $\mathrm{n}^{\mathrm{F}}$ off stack

13 sto $4 \quad 444 \quad$ Store $\mathrm{m}^{\mathrm{F}}$ in register 4

$14 \mathrm{r} \downarrow \quad 33 \quad$ Roll $\mathrm{m}^{\mathrm{F}}$ off stack

15 sto $3 \quad 443 \quad$ Store $1^{\mathrm{F}}$ in register 3

$16 \mathrm{~g}$ rtn $4332 \quad$ Return -- end of program

17 f $1 \mathrm{~b} 1 \mathrm{~d} \quad 422114$ Program D: for hanging-wa11-up faults, reverse the slip direction entered as trend, $T$, and plunge, $P$

$\begin{array}{llll}18 & \text { chs } & 16 & \text { Change sign }: \mathrm{P}=-\mathrm{P} \\ 19 & \mathrm{x}+\mathrm{y} & 34 & \text { Reverse contents of } \mathrm{x} \text { and } \mathrm{y} \text { stack registers } \\ 20 & 1 & 1 & 1 \\ 21 & 8 & 8 & 8 \\ 22 & 0 & 0 & 0 \\ 23 & + & 40 & \mathrm{~T}=180^{\circ}+\mathrm{T} \\ 24 & \mathrm{x}+\mathrm{y} & 34 & \text { Reverse contents of } \mathrm{x} \text { and } \mathrm{y} \text { stack registers }\end{array}$


STEP KEY ENTRY KEY CODE COMMENTS

$25 \mathrm{f}$ fb1 c 422113 Program $\mathrm{c}$ : direction cosines, 1 , $m$ and $n$, of slip direction, $S$, calculated from trend and plunge

$\begin{array}{llll}26 & \text { gsb } 2 & 322 & \text { Go to subroutine } 2 \\ 27 & \text { sto } 8 & 448 & \text { Store } \mathrm{n}^{\mathrm{S}} \text { in register } 8 \\ 28 & \mathrm{r} \downarrow & 33 & \text { Roll } \mathrm{n}^{\mathrm{S}} \text { off stack } \\ 29 & \text { sto } 7 & 447 & \text { Store } \mathrm{m}^{\mathrm{S}} \text { in register } 7 \\ 30 & \text { r } \downarrow & 33 & \text { Roll } \mathrm{m}^{\mathrm{S}} \text { off stack } \\ 31 & \text { sto } 6 & 446 & \text { Store } 1^{\mathrm{S}} \text { in register } 6 \\ 32 & \text { g rtn } & 4332 & \text { Return -- end of program }\end{array}$

33 f 1 bl e 422115 Program E: calculate $\phi$ and $\theta$

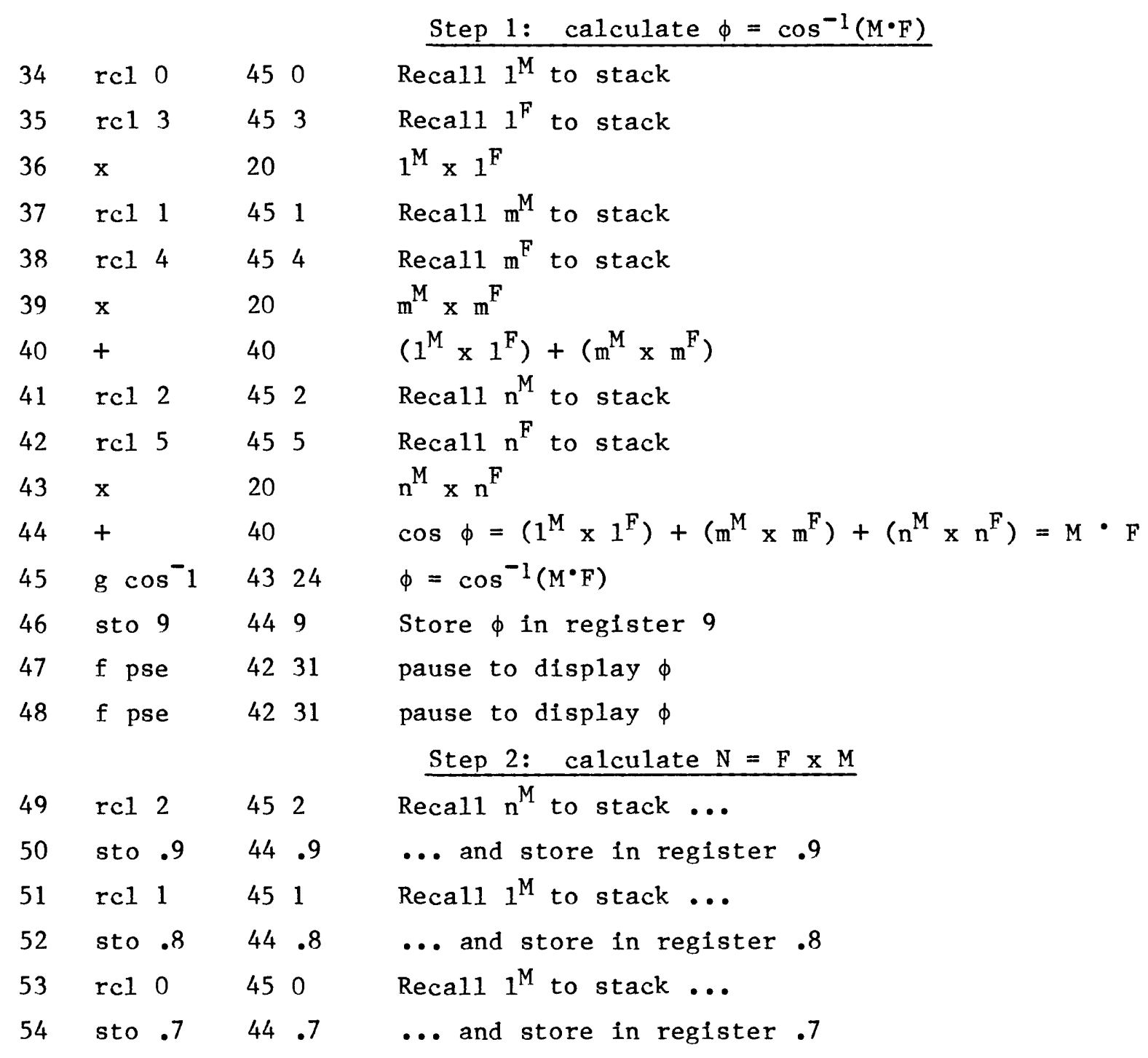




\begin{tabular}{|c|c|c|c|c|}
\hline 55 & $\operatorname{rcl} 5$ & 45 & 5 & Reca11 $\mathrm{n}^{\mathrm{F}}$ to stack \\
\hline 56 & $\operatorname{rcl} 4$ & 45 & 4 & Recall $\mathrm{m}^{\mathrm{F}}$ to stack \\
\hline 57 & rc1 3 & 45 & 3 & Recall $1^{\mathrm{F}}$ to stack \\
\hline 58 & gsb 3 & 32 & 3 & Go to subroutine 3 \\
\hline 59 & sto .3 & 44 & .3 & Store $\mathrm{n}^{\mathrm{N}}$ in register .3 \\
\hline 60 & $r \downarrow$ & 33 & & Roll $n^{N}$ off stack \\
\hline 61 & sto .2 & 44 & .2 & Store $\mathrm{m}^{\mathrm{N}}$ in register .2 \\
\hline 62 & $r \downarrow$ & 33 & & Store Roll $\mathrm{m}^{\mathrm{N}}$ off stack \\
\hline 63 & sto .1 & 44 & .1 & $\begin{array}{l}\text { Store } 1^{N} \text { in register } .1 \\
\text { Step 3: calculate }|\theta|=\cos ^{-1}(|N \cdot S|)\end{array}$ \\
\hline 64 & $\operatorname{rc1} 6$ & 45 & 6 & Recall $1^{\mathrm{S}}$ to stack \\
\hline 65 & $\mathrm{x}$ & 20 & & $1^{\mathrm{N}} \times 1^{\mathrm{S}}$ \\
\hline 66 & $\mathrm{rcl} \quad .2$ & 45 & .2 & Recall $\mathrm{m}^{\mathrm{N}}$ to stack \\
\hline 67 & $\operatorname{rc1} 7$ & 45 & 7 & Recall $\mathrm{m}^{\mathrm{S}}$ to stack \\
\hline 68 & $\mathrm{x}$ & 20 & & $\mathrm{~m}^{\mathrm{N}} \times \mathrm{m}^{\mathrm{S}}$ \\
\hline 69 & + & 40 & & $\left(1^{N} \times 1^{S}\right)+\left(m^{N} \times m^{S}\right)$ \\
\hline 70 & $\operatorname{rcl} \cdot 3$ & 45 & .3 & Recall $\mathrm{n}^{\mathrm{N}}$ to stack \\
\hline 71 & $\operatorname{rc1} 8$ & 45 & 8 & Recall $\mathrm{n}^{\mathrm{S}}$ to stack \\
\hline 72 & $\mathrm{x}$ & 20 & & $\mathrm{n}^{\mathrm{N}} \times \mathrm{n}^{\mathrm{S}}$ \\
\hline 73 & + & 40 & & $\cos \theta=\left(1^{N} \times 1^{S}\right)+\left(m^{N} \times m^{S}\right)+\left(n^{N} \times n^{S}\right)=M \cdot F$ \\
\hline 74 & $g$ test 2 & 43 & 302 & If $\cos \theta<0$ \\
\hline 75 & chs & 16 & & Change sign: $\cos \theta=-\cos \theta$ \\
\hline 76 & $g \cos ^{1}$ & 43 & 24 & $|\theta|=\cos ^{-1}(|N \cdot S|)$ \\
\hline 77 & sto .0 & 44 & .0 & $\begin{array}{l}\text { Store }|\theta| \text { in register } .0 \\
\text { Step 4: If }|\theta|<1^{\circ} \text {, then null fault }\end{array}$ \\
\hline 78 & 1 & 1 & & 1 \\
\hline 79 & - & 30 & & $|\theta|-1$ \\
\hline 80 & $g$ test 1 & 43 & 301 & If $|\theta|-1>0^{\circ}$ \\
\hline 81 & gto 4 & 22 & 4 & Go to label 4 \\
\hline 82 & rel .0 & 45 & .0 & Recall $|\theta|$ to the stack \\
\hline 83 & g rtn & 43 & 32 & $\begin{array}{l}\text { Return - end of program } \\
\text { Step 5: find sign of } \theta \text { using } \mathrm{F} \cdot(\mathrm{NxS})\end{array}$ \\
\hline 84 & f $1 \mathrm{bl} 4$ & 42 & 214 & Labe1 4 \\
\hline & $\operatorname{rc1} 8$ & 45 & 8 & Recall $\mathrm{n}^{\mathrm{S}}$ to stack ... \\
\hline
\end{tabular}




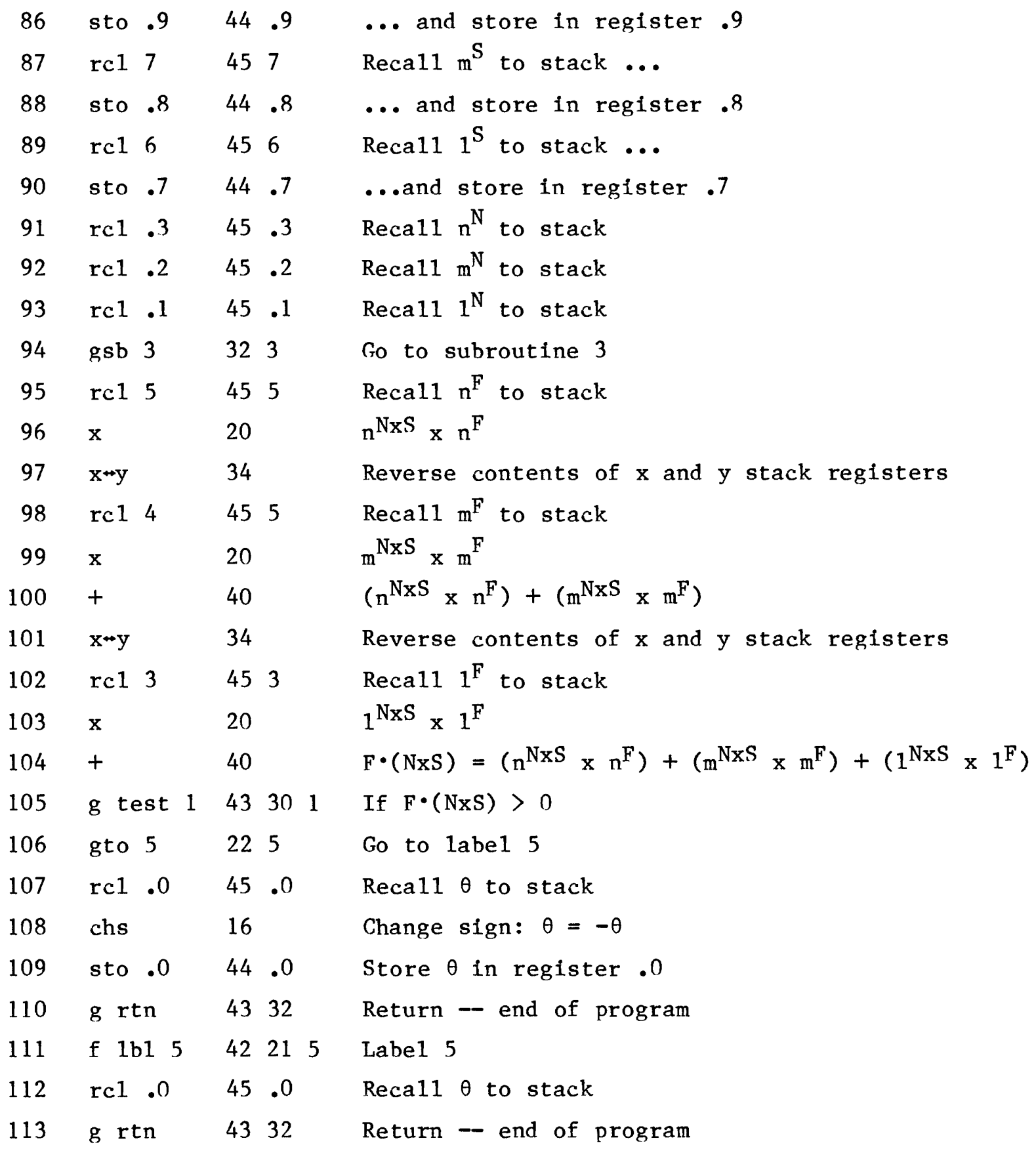

114 f 1 b1 $1 \quad 4221 \quad 1$ Subroutine 1: calculate direction cosines, 1 , m, and $n$, from dip direction, $D D$, and dip, $D$

115 sto $.9 \quad 44.9 \quad$ Store D in register .9

$116 \sin 23 \quad$ sin $D$

$117 \quad \mathrm{x} \bullet \mathrm{y} \quad 34 \quad$ Reverse contents of $\mathrm{x}$ and $\mathrm{y}$ stack registers 
STEP KEY ENTRY KEY CODE COMMENTS

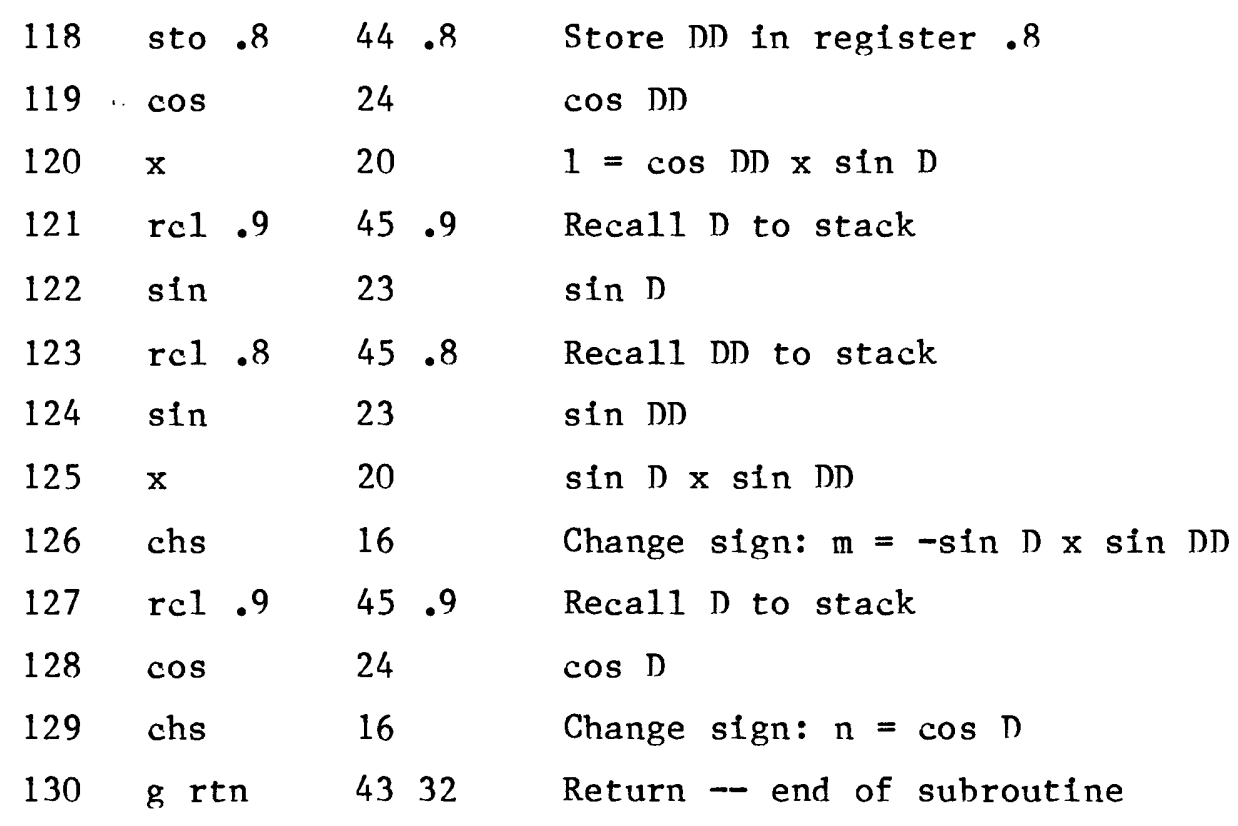

131 f $1 \mathrm{~b} 1242212$ Subroutine 2: Calculate direction cosines, 1 , $m$ and $n$, from trend, $T$, and plunge, $p$

\begin{tabular}{|c|c|c|c|c|c|}
\hline 132 & sto .9 & 9 & 44 & .9 & Store $\mathrm{P}$ in register .9 \\
\hline 133 & $\cos$ & & 24 & & $\cos \mathrm{P}$ \\
\hline 134 & $x \leftrightarrow y$ & & 34 & & Reverse contents of $\mathrm{x}$ and $\mathrm{y}$ stack registers \\
\hline 135 & sto $\cdot 8$ & 8 & 44 & .8 & Store $\mathrm{T}$ in register .8 \\
\hline 136 & $\cos$ & & 24 & & $\cos T$ \\
\hline 137 & $\mathrm{x}$ & & 20 & & $\cos T \times \cos P$ \\
\hline 138 & chs & & 16 & & Change sign: $1=-\cos T \times \cos P$ \\
\hline 139 & $\mathrm{rel} .9$ & 9 & 45 & .9 & Recal1 P to stack \\
\hline 140 & $\cos$ & & 24 & & $\cos P$ \\
\hline 141 & $\mathrm{rcl} \cdot 8$ & 8 & 45 & .8 & Recall $\mathrm{T}$ to stack \\
\hline 142 & $\sin$ & & 23 & & $\sin T$ \\
\hline 143 & $\mathrm{x}$ & & 20 & & $\mathrm{~m}=\sin \mathrm{T} \times \cos \mathrm{P}$ \\
\hline 144 & rel .9 & 9 & 45 & .9 & Recall P to stack \\
\hline 145 & $\sin$ & & 23 & & $\sin P$ \\
\hline 146 & chs & & 16 & & Change sign: $n=-\sin P$ \\
\hline 147 & g rtn & & 43 & 32 & Return - end of subroutine \\
\hline
\end{tabular}


148 f 1 bl 342213 Subroutine 3: calculate and normalize to unit length the cross product $A \times B$

Step 1: $\mathrm{C}=\mathrm{A} \times \mathrm{X}$

149 sto $.4 \quad 44.4 \quad$ Store $1^{\mathrm{A}}$ in register .4

$150 \mathrm{r} \downarrow \quad 33 \quad$ Roll $1^{\mathrm{A}}$ off stack

151 sto $.5 \quad 44.5 \quad$ Store $\mathrm{m}^{\mathrm{A}}$ in register .5

$152 \quad \mathrm{r} \downarrow \quad 33 \quad$ Roll $\mathrm{m}^{\mathrm{A}}$ of $\mathrm{f}$ stack

153 sto $.6 \quad 44.6 \quad$ Store $\mathrm{n}^{\mathrm{A}}$ in register .6

$\begin{array}{llllll}154 & \mathrm{rcl} & .5 & 45 & .5 & \operatorname{Recall} \mathrm{m}^{\mathrm{A}} \text { to stack }\end{array}$

$155 \quad \operatorname{rcl} .9 \quad 45.9 \quad \operatorname{Recall} \mathrm{n}^{\mathrm{B}}$ to stack

$156 \times 20 \quad \mathrm{~m}^{\mathrm{A}} \times \mathrm{n}^{\mathrm{B}}$

$\begin{array}{llllll}157 & \mathrm{rcl} & .8 & 45 & .8 & \text { Recall } \mathrm{m}^{\mathrm{B}} \text { to stack }\end{array}$

$\begin{array}{llllll}158 & \operatorname{rcl} & .6 & 45.6 & \operatorname{Reca} 11 \mathrm{n}^{\mathrm{A}} \text { to stack }\end{array}$

$159 \times 20 \quad n^{A} \times m^{B}$

$160 \quad 30 \quad 1^{\mathrm{C}}=\left(\mathrm{n}^{\mathrm{A}} \times \mathrm{m}^{\mathrm{B}}\right)-\left(\mathrm{m}^{\mathrm{A}} \times \mathrm{n}^{\mathrm{B}}\right)$

161 sto $.1 \quad 44.1 \quad$ Store $1^{C}$ in register .1

$\begin{array}{llllll}162 & \operatorname{rcl} & .7 & 45 & .7 & \text { Recall } 1^{\mathrm{B}} \text { to stack }\end{array}$

$163 \mathrm{rcl} .6 \quad 45.6 \quad$ Recall $\mathrm{n}^{\mathrm{A}}$ to stack

$164 \times 20 \quad \mathrm{n}^{\mathrm{A}} \times 1^{\mathrm{B}}$

$\begin{array}{lllllll}165 & \mathrm{rcl} & .4 & 45 & .4 & \text { Recall } 1^{\mathrm{A}} \text { to stack }\end{array}$

$166 \quad \mathrm{rcl} .9 \quad 45.9 \quad$ Recall $\mathrm{n}^{\mathrm{B}}$ to stack

$167 \times 20 \quad n^{B} \times 1^{A}$

$168-30 \quad m^{\mathrm{C}}=\left(\mathrm{n}^{\mathrm{B}} \times 1^{\mathrm{A}}\right)-\left(\mathrm{n}^{\mathrm{A}} \times 1^{\mathrm{B}}\right)$

169 sto $.2 \quad 44.2 \quad$ Store $\mathrm{m}^{\mathrm{C}}$ in register .2

$\begin{array}{llllll}170 & \mathrm{rcl} & .4 & 45 & .4 & \operatorname{Recal} 11^{\mathrm{A}} \text { to stack }\end{array}$

$\begin{array}{lllllll}171 & \mathrm{rcl} & .8 & 45 & .8 & \text { Recall } \mathrm{m}^{\mathrm{B}} \text { to stack }\end{array}$

$172 \times \quad 20 \quad \mathrm{~m}^{\mathrm{B}} \times 1^{\mathrm{A}}$

$\begin{array}{llllll}173 & \operatorname{rcl} & .7 & 45 & .7 & \text { Recall } 1^{\mathrm{B}} \text { to stack }\end{array}$

$174 \quad \operatorname{rc} 1.5 \quad 45.5 \quad \operatorname{Recal1} \mathrm{m}^{\mathrm{A}}$ to stack

$175 \times 20 \quad \mathrm{~m}^{\mathrm{A}} \times 1^{\mathrm{B}}$

$176 \quad 30 \quad n^{C}=\left(m^{A} \times 1^{B}\right)-\left(m^{B}-1^{A}\right)$

177 sto $.3 \quad 44.3 \quad$ Store $\mathrm{n}^{\mathrm{C}}$ in register .3

$178 \quad \mathrm{gx}^{2} \quad 4311 \quad\left(\mathrm{n}^{\mathrm{C}}\right)^{2}$

Step 2: $\quad D=C / \sqrt{C \cdot C}$ 
STEP KEY ENTRY KEY CODE COMMENTS

\begin{tabular}{|c|c|c|c|c|}
\hline 179 & $\operatorname{rcl} \cdot 2$ & 45 & .2 & Recal1 $\mathrm{m}^{\mathrm{C}}$ to stack \\
\hline 180 & $g x^{2}$ & 43 & 11 & $\left(m^{C}\right)^{2}$ \\
\hline 181 & + & 40 & & $\left(m^{C}\right)^{2}+\left(n^{C}\right)^{2}$ \\
\hline 182 & $\operatorname{rc1} .1$ & 45 & .1 & Recal1 $1^{\mathrm{C}}$ to stack \\
\hline 183 & $g x^{2}$ & 43 & 11 & $\left(1^{C}\right)^{2}$ \\
\hline 184 & + & 40 & & $C \cdot C=\left(1^{C}\right)^{2}+\left(m^{C}\right)^{2}+\left(n^{C}\right)^{2}$ \\
\hline 185 & $\sqrt{ }$ & 11 & & $\sqrt{C \cdot C}$ \\
\hline 186 & sto .9 & 44 & .9 & Store $\sqrt{C \cdot C}$ in register .9 \\
\hline 187 & $\operatorname{rcl} \cdot 1$ & 45 & .1 & Recal1 $1^{\mathrm{C}}$ to stack \\
\hline 188 & $\operatorname{rcl} .9$ & 45 & .9 & Recall $\sqrt{\mathrm{C} \cdot \mathrm{C}}$ to stack \\
\hline 189 & $\div$ & 10 & & $1^{D}=1^{C} / \sqrt{C \cdot C}$ \\
\hline 190 & $\mathrm{rcl} \cdot 2$ & 45 & .2 & Reca $11 \mathrm{~m}^{\mathrm{C}}$ to stack \\
\hline 191 & $\operatorname{rcl} .9$ & 45 & .9 & Recall $\sqrt{C \cdot C}$ to stack \\
\hline 192 & $\div$ & 10 & & $\mathrm{~m}^{\mathrm{D}}=\mathrm{m}^{\mathrm{C}} / \sqrt{\mathrm{C} \cdot \mathrm{C}}$ \\
\hline 193 & $\mathrm{rcl} \cdot 3$ & 45 & .3 & Recall $\mathrm{n}^{\mathrm{C}}$ to stack \\
\hline 194 & $\operatorname{rc1} \quad .9$ & 45 & .9 & Recall $\sqrt{C \cdot C}$ to stack \\
\hline 195 & $\div$ & 10 & & $\mathrm{n}^{\mathrm{D}}=\mathrm{n}^{\mathrm{C}} / \sqrt{\mathrm{C} \cdot \mathrm{C}}$ \\
\hline 196 & $g \operatorname{rtn}$ & 43 & 32 & Return - end of subroutine \\
\hline
\end{tabular}

